\title{
Emergency Surgical Intervention to Treat a Wide Septated Subdural Hematoma in a Patient with ITP: A Case Report
}

\author{
Ziya Asan' ${ }^{\circ}$, Asuman Kilitci ${ }^{\circledR}$ \\ 'Department of Neurosurgery, Ahi Evran University School of Medicine, Kırșehir, Turkey \\ ${ }^{2}$ Department of Pathology, Ahi Evran University School of Medicine, Kırșehir, Turkey
}

ORCID IDs of the authors: Z.A. 0000-000I-8468-9I56; A.K. 0000-0002-5489-2222.

Cite this article as: Asan Z, Kilitci A. Emergency Surgical Intervention to Treat a Wide Septated Subdural Hematoma in a Patient with ITP: A Case Report. Cyprus J Med Sci 2019; 4(I): 63-5.

\begin{abstract}
Immune thrombocytopenic purpura (ITP) is a syndrome that reduces platelet levels, and it is caused by the development of autoantibodies against thrombocytes. The ITP cases that present with subdural hematoma are rarely seen. It is necessary to start corticosteroid and intravenous immunoglobulin (IVIg) treatment early in the emergency cases, until a definite diagnosis is made. A 54-year-old female patient applied to the neurosurgery department with the complaint of dizziness that lasted for a week. The patient indicated that she had an upper respiratory tract infection a month ago. On her computed tomography examination, she was diagnosed with a large and septated subdural hematoma with different components of acute, subacuteand chronic bleeding with the ITP diagnosis. The patient had to undergo an urgent surgical procedure due to right hemiparesis and confusion caused by the finding of increased intracranial pressure. During pharmacotherapy for ITP, the patient was re-operated due to recurrent bleeding. The patient was discharged without any deficiency after the second operation. Thrombocytopenia cases are challenging with regard to surgical intervention. It is necessary to start corticosteroid treatment early in emergency cases, until the definite diagnosis is made. Corticosteroids inhibit the development of autoantibodies in the treatment of ITP but also increase the release of platelets from the vascular wall. Corticosteroid treatment together with IVlg treatment should be initiated, and in the case of an emergency surgical intervention, a thrombocyte replacement needs to be initiated immediately.
\end{abstract}

Keywords: Septated subdural hematoma, immune thrombocytopenic, purpura thrombocytopenia, multicomponent subdural hematoma

\section{INTRODUCTION}

Immune thrombocytopenic purpura (ITP) is a syndrome that reduces platelet levels, and it is caused by the development of autoantibodies against thrombocytes. The ITP cases that present with subdural hematoma are rarely seen (I). It is necessary to start corticosteroid and intravenous immunoglobulin (IVIg) treatment early in emergency cases, until a definite diagnosis is made $(2,3)$. In this paper, we aimed to present a wide septated subdural hematoma that occurred in a case diagnosed with ITP and an emergency surgical intervention.

\section{CASE PRESENTATION}

A 54-year-old female patient was admitted to the hospital complaining of dizziness that lasted for a week. The patient did not have any complaints of headache, vomiting, epileptic seizures, and neurological deficit; however, purpural rash was observed on the examination and was more prominent in the upper limbs. Frust hemiparesia was present on the right side on the neurological examination. Bilateral papillary edema was detected in the fundus oculi upon the ophthalmic examination. The patient indicated that she had an upper respiratory tract infection a month ago and that her blood test results were as follows: hemoglobin, II.4; leucocytes, 8500; and the platelet value, 5000. The prothrombin time (PT) and activated partial thromboplastin time (aPTT) were determined, respectively, as 12 and 29. Liver and kidney function tests were within the normal limits. Splenomegaly was not detected upon an abdominal ultrasound examination. Serologic tests were negative. Peripheral smear was compatible with thrombocytopenia, and atypical cells were not detected. The ANA (hep2) and Coombs tests were negative. The patient did not mention any use of antiaggregants or anticoagulants.

The cranial magnetic resonance imaging (MRI) examination showed a subdural hematoma in the left fronto-parietal area, which was $6 \mathrm{~cm}$ thick in the largest part, was causing a 2-cm-thick midline shift, and also contained a large number 
of septations (Figure I). An underlying vascular pathology was not detected in the MR angiography. The patient was given intravenous methylprednisolone I g/day and IVIg (I g/ kg/day) as treatment. One day later, the number of platelets increased to II.000 in control blood tests. On the same day, the patient had to undergo an urgent surgical procedure due to right hemiparesis and confusion caused by the finding of increased intracranial pressure. The preoperative Glasgow coma scale was evaluated as $10 / 15$.
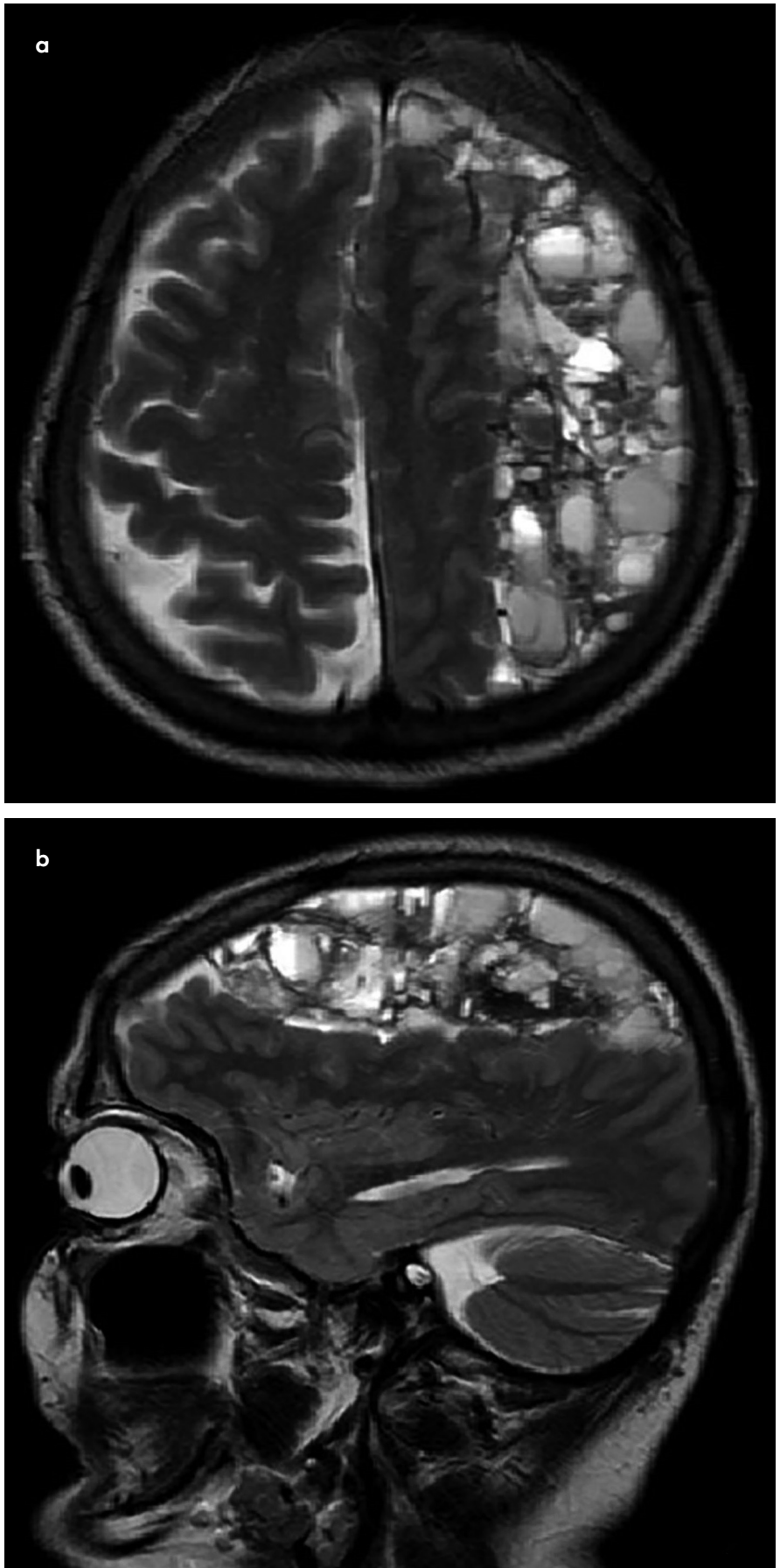

FIGURE I. a, b. In the magnetic resonance imaging (MRI) examination, a wide septated subdural hematoma with different components can be seen (a) in the left fronto-parietal area, $6 \mathrm{~cm}$ thick in the largest part, causing a 2-cm-thick midline shift, and also containing a large number of septations (b)
The patient was operated with left fronto-parietal craniotomy, and subdural hematoma was evacuated. Levetiracetam treatment was started as an antiepileptic treatment in the postoperative period. The platelet count was 18.000 on the first postoperative day, and the corticosteroid treatment continued. The patient was re-operated upon the findings of increased intra-
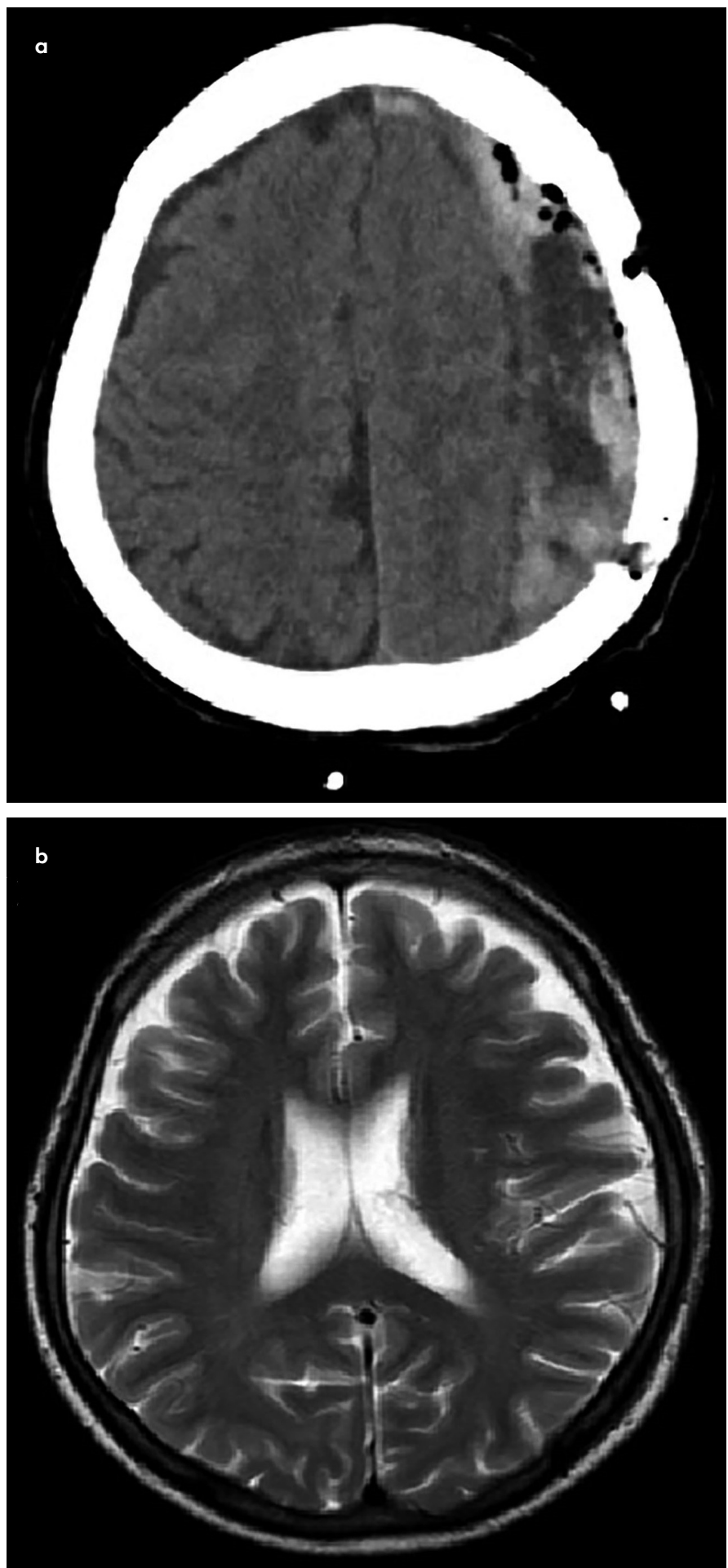

FIGURE 2. a, b. On the postoperative 3rd day computed tomography $(\mathrm{CT})$ scan, it can be seen that the bleeding occurred again in the operation area (a). On the MR examination 5 months after the operation, it can be seen that the subdural space was completely closed, and there were no pathologies that could lead to spontaneous bleeding (b) 
cranial pressure, and hemorrhage was detected again in the same region on the computed tomography (CT) scan (Figure 2a). During surgery, the acute subdural hematoma in the same region was evacuated. No additional neurological deficits were detected in the postoperative early period, except the right frust hemiparesis. On the fifth postoperative day, the patient's platelet count was 23.000, and the patient was discharged without any neurological deficits 8 days after the second operation, because the cranial CT revealed that the blood in the subdural space was reabsorbed. Eight months after the operation, the $\mathrm{MRI}$ examinations revealed that the subdural space was completely closed and that there was no any other pathological formation (Figure 2b). Informed consent form was obtained from the patient.

\section{DISCUSSION}

Immune thrombocytopenic purpura is a syndrome that reduces platelet level sand it is caused by the development of autoantibodies against thrombocytes. Coagulation parameters are within the normal range. The most serious and life-threatening complication of ITP is intracranial hemorrhage (I). A reduced platelet count is a risk factor for the development of spontaneous intracranial hemorrhage, and in addition, minor traumas can lead to intracranial hemorrhages.

In the case of ITP, the PT and aPTT values are within the normal limits because there is no factor deficiency. Bleeding and coagulation times are prolonged due to a low platelet count. Therefore, there is no efficacy of plasma replacement when surgical intervention is to be applied to ITP cases.

For these cases, corticosteroid treatment is administered to stop the development of autoantibodies (2). Sole thrombocyte replacement is not curable because the autoantibody presence persists. A continued autoantibodies presence leads to the destruction of replaced platelets. Therefore, corticosteroid and IVIg treatment is applied to prevent the autoantibody development during the treatment (2). Corticosteroids are the first drug of choice in the treatment; however, if the use of corticosteroids is contraindicated and if an emergency intervention is needed, $\mathrm{IVIg}$ is preferred. However, the efficacy of the IVIg treatment is temporary and lasts for 2-4 weeks (3).

Subdural hematomas are the most commonly seen intracranial hemorrhages; however, subdural hematoma cases caused by ITP are rarely seen. A low platelet level is a risk factor for the subdural hematoma formation, but it is not associated with an increase in the hematoma size (I). It is thought that the hematoma volume increases due to the fibrinolytic activity in hematomas that are larger in volume and that were early asymptomatic and were detected in the chronic phase.

Septated subdural hematomas are rarely seen. The presence of a large number of septations and the presence of acute, sub- acute, and chronic components of the hematoma at the same time suggest that the bleeding repeated many times at different times. Due to the presence of numerous septations, the craniotomy method was preferred during surgery.

Timing of the surgical treatment is vital in ITP cases. The platelet count needs to be at the acceptable levels as much as possible in surgical timing. In these cases, the number of platelets lower than 20,000 is a treatment indication (I). The main goal of treatment in adult ITP is to achieve a safe number of platelets, and not to completely normalize the platelet value.

A thrombocyte replacement solely may not be enough in cases that need an emergency surgical intervention. The main underlying pathology is the presence of autoantibodies, and thrombocyte replacement is not enough alone to increase the platelet count due to the presence of autoantibodies (I). For this reason, a platelet replacement together with IVIg and corticosteroid treatments should be performed (I).

Thrombocytopenia cases are challenging for surgical intervention. It is necessary to start the corticosteroid treatment early in the emergency cases until definite diagnosis is made. Corticosteroids inhibit the development of autoantibodies in the treatment of ITP, but also increase the release of platelets from the vascular wall. Corticosteroid treatment together with IVIg treatment should be initiated, and in emergency surgical interventions, thrombocyte replacement needs to be initiated immediately.

Informed Consent: Written informed consent was obtained from patients who participated in this study.

Peer-review: Externally peer-reviewed.

Author contributions: Concept - A.K., Z.A.; Design - A.K., Z.A.; Supervision - A.K., Z.A.; Resource - Z.A.; Materials - A.K., Z.A.; Data Collection and/or Processing - Z.A., A.K.; Analysis and/or Interpretation - A.K., Z.A.; Literature Search - Z.A.; Writing - Z.A., A.K.; Critical Reviews - Z.A., A.K.

Conflict of Interest: The authors have no conflicts of interest to declare.

Financial Disclosure: The authors declared that this study has received no financial support.

\section{REFERENCES}

I. Chatteriee S, Karmakar PS, Ghosh P, Ghosh A. Subdural hematoma associated with immune thrombocytopenic purpura in two different clinical settings. J Assoc Physicians India 20I0; 58: 504-6.

2. Neunert C, Lim W, Crowther M, Cohen A, Solberg L Jr, Crowther MA. The American Society of Hematology 2011 evidence-based practice guideline for immune thrombocytopenia. Blood 20Il; II7: 4190-207. [CrossRef]

3. Rodeghiero F, Schiavotto C, Castaman G, Vespignani M, Ruggeri M, Dini E. A follow-up study of 49 adult patients with idiopathic thrombocytopenic purpura treated with high-dose immunoglobulins and anti-D immunoglobulins. Haematologica 1992; 77: 248-52. 